Following publication of the Research Article by Jessica Schiavi, Laetitia Keller, David-Nicolas Morand, Natalia De Isla, Olivier Huck, Jean Christophe Lutz, Didier Mainard, Pascale Schwinté \& Nadia Benkirane-Jessel, titled 'Active implant combining human stem cell microtissues and growth factors for bone-regenerative nanomedicine', which appeared in the March 2015 issue of Nanomedicine (Nanomedicine [Lond.]) 10 [5], 753-763 [2015]), it has been brought to our attention that the first three paragraphs on page 755 were presented incorrectly as:

\section{Chondrogenic differentiation}

hMSCs were seeded as a pellet of 250,000 cells and cultured for 28 days with standard chondrogenic medium (Lonza, France) supplemented with $10 \mathrm{ng}$ ml-1 of TGF- $\beta 3$. Samples were then fixed with $4 \%$ formalde cartilage glycosaminoglycans.

Osteogenic differenti hyde and paraffin sections were obtained and stained with Alcian Blue staining to visualization.

hMSCs were seeded at 100 cells $\mathrm{cm}-2$ and put in culture for 14 days with hMSCs medium. After that, we induced differentiation by supplementing standard culture medium with ascorbic acid $60 \mu \mathrm{m}, \beta$-glycerol phosphate $10 \mathrm{~mm}$ and dexamethasone $0.1 \mu \mathrm{m}$ for 21 days. Cells were washed with PBS, fixed in ice-cold 70\% ethanol and stained with Alizarin Red (pH: 4.1; Sigma-Aldrich, France) to detect $\mathrm{Ca} 2+$ deposits.

The correct presentation should be:

\section{Chondrogenic differentiation}

hMSCs were seeded as a pellet of 250,000 cells and cultured for 28 days with standard chondrogenic medium (Lonza, France) supplemented with $10 \mathrm{ng} \mathrm{ml}^{-1}$ of TGF- $\beta_{3}$. Samples were then fixed with $4 \%$ formaldehyde and paraffin sections were obtained and stained with Alcian Blue staining to visualize cartilage glycosaminoglycans.

\section{Osteogenic differentiation}

hMSCs were seeded at 100 cells cm$~^{-2}$ and put in culture for 14 days with hMSCs medium. After that, we induced differentiation by supplementing standard culture medium with ascorbic acid $60 \mu \mathrm{m}, \beta$-glycerol phosphate $10 \mathrm{~mm}$ and dexamethasone $0.1 \mu \mathrm{m}$ for 21 days. Cells were washed with PBS, fixed in ice-cold 70\% ethanol and stained with Alizarin Red (pH: 4.1; Sigma-Aldrich, France) to detect $\mathrm{Ca}^{2+}$ deposits.

The editors of Nanomedicine would like to sincerely apologize for any inconvenience or confusion this may have caused our readers. 